\title{
Downregulation of NIT2 inhibits colon cancer cell proliferation and induces cell cycle arrest through the caspase-3 and PARP pathways
}

\author{
BO'AN ZHENG $^{1 *}$, RUI CHAI ${ }^{*}$ and XIAOJUN YU ${ }^{2}$ \\ Departments of ${ }^{1}$ Colorectal Surgery, and ${ }^{2}$ Gastroenterological Surgery, \\ Zhejiang Provincial People's Hospital, Hangzhou, Zhejiang 310014, P.R. China
}

Received May 13, 2014; Accepted December 30, 2014

DOI: $10.3892 /$ ijmm.2015.2125

\begin{abstract}
Colorectal cancer, also known as colon cancer is the most devastating malignancy worldwide. Previous studies have reported that NIT2, a member of the nitrilase superfamily, is a potential tumor suppressor, although its function remains elusive in colon cancer. In the present study, we employed an RNA interference lentivirus system to silence endogenous NIT2 expression in the colon cancer cell line, HCT116. The knockdown efficiency was determined by RT-qPCR and western blot analysis. The depletion of NIT2 markedly inhibited colon cancer cell proliferation and colony formation and induced cell cycle arrest in the G0/G1 phase, as shown by MTT assay, colony fromation assay and flow cytometric analysis, respectively. Further investigation with an intracellular signaling array demonstrated that the depletion of NIT2 triggered the apoptosis of colon cancer cells through the caspase- 3 and poly(ADP-ribose) polymerase (PARP) pathways. Our findings suggest that NIT2 may be an oncogene in human colon cancer and may thus serve as a promising therapeutic target for the treatment of colon cancer.
\end{abstract}

\section{Introduction}

Colorectal cancer, also known as colon cancer, is the most common type of gastrointestinal cancer worldwide. Despite advances in research and treatment modalities, colorectal cancer still causes 500,000 deaths each year (1), and approximately 150,000 US residents are diagnosed annually with colon cancer (2). It is one of the devastating cancers and one

Correspondence to: Professor Xiaojun Yu, Department of Gastroenterological Surgery, Zhejiang Provincial People's Hospital, 158 Shangtang Road, Hangzhou, Zhejiang 310014, P.R. China E-mail: xiaojunyudr@163.com

${ }^{*}$ Contributed equally

Key words: colon cancer, NIT2, RNA interference, proliferation third of patients with colon cancer will eventually succumb to the disease (3). Over the past 3 decades, investigations into the molecular genetics of colon cancer have revealed the regulation of cellular metabolism, proliferation, differentiation and survival in colon cancer (4). However, much work remains to be done in order to fully understand and integrate the molecular changes associated with the pathophysiology of colorectal cancer.

Human nitrilase (NIT) proteins are members of the nitrilase superfamily that contain a conserved nitrilase domain and thiol enzymes involved in natural product biosynthesis and post-translational modification in plants (5), animals, fungi and certain prokaryotes $(6,7)$. Based on the sequence analysis, the NIT superfamily has been divided into 13 branches $(6,8)$. Branch 10 in the Brenner classification contains only 2 enzymes, designated as NIT1 and NIT2, found in mammalian tissues (8-10). The NIT2 gene is ubiquitously expressed in multiple types of tissue and encodes the protein NIT2 mainly distributed in the cytosol; it has been reported that NIT2 is a potential tumor suppressor (11). However, to the best of our knowledge, no study to date has reported the role of NIT2 in colon cancer, and the cellular function of the NIT2 gene remains elusive.

The RNA interference (RNAi) technique is a powerful tool to carry out loss-of-function assays. It provides a new approach to investigating cancer gene therapy $(12,13)$. In the present study, we employed a lentivirus-mediated RNAi system in order to achieve the highly stable silencing of NIT2 in the colon cancer cell line, HCT116. We evaluated the biological function of NIT2 and aimed to reveal its contribution to the progression of colon cancer. To the best of our knowledge, this is the first presentation providing evidence that the knockdown of endogenous NIT2 expression suppresses the oncogenic properties of colon cells and induces apoptosis through the caspase- 3 and poly(ADP-ribose) polymerase (PARP) pathways.

\section{Materials and methods}

Cell culture. The human embryonic kidney cell line 293T (HEK293T) and the human colon cancer cell line, HCT116, were obtained from the Shanghai Institute of Cell Biology, the Chinese Academy of Sciences, Shanghai, China. The HCT116 cells were maintained in McCoy's 5A medium (Sigma-Aldrich, St. Louis, MO, USA) supplemented with $10 \%$ fetal bovine serum (FBS) 
at $37^{\circ} \mathrm{C}$ in a humidified atmosphere of $5 \% \mathrm{CO}_{2}$. The HEK293T cells were maintained in Dulbecco's modified Eagle's medium (DMEM) (HyClone, Logan, UT, USA) supplemented with $10 \%$ FBS at $37^{\circ} \mathrm{C}$ in a humidified atmosphere of $5 \% \mathrm{CO}_{2}$.

Construction of recombinant lentivirus. The following oligonucleotides were synthesized. The short hairpin RNA (shRNA) sequence (5'-ACATAATCAACTCCCTATTAACT CGAGTTAATAGGGAGTTGATTATGTTTTTT-3', sequence 1) against the human NIT2 gene (NM_020202) was screened and validated to be a candidate shRNA. The negative control shRNA was 5'-TTCTCCGAACGTGTCACGT-3'. The stem-loop-stem oligos (shRNAs) were synthesized, annealed and ligated into the AgeI/EcoRI-linearized pFH-L vector (Shanghai Hollybio, Shanghai, China). The lentiviral-based shRNA-expressing vectors were confirmed by DNA sequencing. The generated plasmids were named pFH-L-shNIT2 and pFH-L-shCon.

The HEK293T cells $\left(1.0 \times 10^{6}\right)$ were seeded into $10-\mathrm{cm}$ dishes and cultured for $24 \mathrm{~h}$ to reach $70-80 \%$ confluence as previously described (14). Two hours prior to transfection, the medium was replaced with serum-free DMEM. The plasmids, including $10 \mu \mathrm{g}$ of pFH-L-shNIT2 or pFH-L-shCon, $7.5 \mu \mathrm{g}$ of the packaging vector pHelper-1.0 and $5 \mu \mathrm{g}$ of the expression plasmid pHelper-2.0 were added to $0.95 \mathrm{ml}$ of Opti-MEM and $50 \mu \mathrm{l}$ of Lipofectamine 2000. The mixture was added to the cells followed by incubation for $8 \mathrm{~h}$ before replacing the medium with $10 \mathrm{ml}$ of complete DMEM medium (supplemented with $10 \%$ PBS). Lentiviral particles were harvested $48 \mathrm{~h}$ after transfection. The HCT116 cells $\left(8.0 \times 10^{4}\right)$ were cultured in 6-well plates and inoculated with recombinant lentiviruses (Lv-shCon or Lv-shNIT2) at a multiplicity of infection (MOI) of 15. As the lentivirus carries green fluorescence protein (GFP), the infection efficiency was determined by counting the numbers of GFP-expressing cells under a fluorescence microscope (Olympus BX50; Olympus Corp., Tokyo, Japan) at $96 \mathrm{~h}$ after infection.

RNA extraction and reverse transcription-quantitative (realtime) PCR (RT-qPCR). The human colon cancer cells, HCT116, were pre-cultured and infected with the recombinant lentiviruses for 5 days as previously described (15). Total RNA was prepared using TRIzol reagent (Gibco BRL, Grand Island, NY, USA) according to the manufacturer's instructions. Total RNA (5 mg) was used to synthesize the first-strand cDNA using $200 \mathrm{U} / \mathrm{ml}$ SuperScript II Reverse Transcriptase (RT) (Invitrogen, Carlsbad, CA, USA). The NIT2 mRNA expression was evaluated by quantitative PCR on a BioRad Connet RealTime PCR platform (Bio-Rad Laboratories, Hercules, CA, USA) with SYBR-Green PCR Core reagents. The qPCR reaction system contains $10 \mu \mathrm{l} 2 \mathrm{X}$ SYBR Premix Ex Taq, plus $0.8 \mu \mathrm{l}$ forward and reverse primers $(2.5 \mu \mathrm{M}), 5 \mu \mathrm{l}$ cDNA template and $4.2 \mu \mathrm{l} \mathrm{ddH_{2 }}$ O. $\beta$-actin was applied as the internal reference. The following primers were synthesized and applied: NIT2 forward, 5'-CGGGCTGTTGATAATCAGGT-3' and reverse, 5'-TTCAGCCAGCTTCTTCAGGT-3'; and $\beta$-actin forward, 5'-GTGGACATCCGCAAAGAC-3' and reverse, 5'-A AAGGGTGTAACGCAACTA-3' (14). The reaction procedure was initiated with denaturation at $95^{\circ} \mathrm{C}$ for $1 \mathrm{~min}$ followed by 40 repeated cycles (denaturation at $95^{\circ} \mathrm{C}$ for $5 \mathrm{sec}$ and annealing extension at $60^{\circ} \mathrm{C}$ for $20 \mathrm{sec}$ ). The results are presented as CT values, defined as the threshold PCR cycle number at which an amplified product is first detected. The average $\mathrm{CT}$ value was calculated for both NIT2 and $\beta$-actin, and the $\Delta \mathrm{CT}$ value was determined as the mean of the triplicate CT values for NIT2 minus the mean of the triplicate CT values for $\beta$-actin.

Western blot analysis. The HCT116 cells were cultured and infected with the recombinant lentiviruses for 5 days. The cells were washed twice with ice-cold PBS and lysed in 2X SDS sample buffer [100 mM Tris- $\mathrm{HCl}$ (pH 6.8), 10 mM EDTA, $4 \%$ SDS and $10 \%$ glycine]. Equal amount of proteins $(30 \mu \mathrm{g})$ were loaded and separated by electrophoresis $(50 \mathrm{~V}, 3 \mathrm{~h})$ on $10 \%$ SDS-PAGE gels and transferred onto polyvinylidene difluoride (PVDF) membranes (Millipore, Bedford, MA, USA) at $300 \mathrm{~mA}$ for $1.5 \mathrm{~h}$. The membranes were blocked and then probed with primary antibodies, rabbit anti-NIT2 $(1: 8,000$ dilution, \#31280; Signalway Antibody LLC, College Park, MD, USA) or mouse anti-GAPDH (1:50,000 dilution, \#10494-1-AP; Santa Cruz Biotechnology, Inc., Dallas, Texas, USA) overnight at $4^{\circ} \mathrm{C}$. After washing, the blots were incubated with horseradish peroxidase-conjugated secondary antibodies (1:5,000 dilution, \#SC-2054; Santa Cruz Biotechnology, Inc.) for $2 \mathrm{~h}$ at room temperature and then visualized using Super ECL Detection reagent (Applygen Technologies, Inc., Beijing, China).

Colony formation assay. The human colon cancer cells, HCT116, were cultured in 6-well plates and treated with the recombinant lentiviruses. After $96 \mathrm{~h}$ of incubation, the infected cells were washed, re-cultured in the prepared 6-well plates at a density of 400 cells/well, and allowed to form natural colonies. After 7 days of incubation, the treated cells were subjected to Crystal violet staining. Subsequently, the cells were washed and fixed with paraformaldehyde. The fixed cells were washed twice with PBS solution, treated with Crystal violet for $10 \mathrm{~min}$, washed 3 times with $\mathrm{dd}_{2} \mathrm{O}$ and then photographed using a digital camera (D7000; Nikon Corp., Tokyo, Japan). The number of colonies ( $>50$ cells/colony) was counted.

MTT assay for cell viability. The HCT116 cells were cultured in 6-well plates and inoculated with the recombinant lentiviruses. After $96 \mathrm{~h}$ of infection, the cells were washed and re-cultured in 96-well plates at $2.5 \times 10^{3}$ cells/well. MTT solution was added to the wells followed by incubation at $37^{\circ} \mathrm{C}$ for $4 \mathrm{~h}$ at different time points after lentivirus infection $(1$, 2, 3, 4 and 5 days). The converted dye was then solubilized in acidic isopropanol (10\% SDS, 5\% isopropanol and $0.01 \mathrm{M}$ $\mathrm{HCl}$ ) followed by incubation at $37^{\circ} \mathrm{C}$ for $10 \mathrm{~min}$. The optical density was measured using a microplate reader (Epoch; BioTek, Winooski, VT, USA) at the wavelength of $595 \mathrm{~nm}$. The experiment was repeated at least 3 times.

Flow cytometric analysis. The HCT116 cells were cultured in 6 -well plates and inoculated with the recombinant lentiviruses at a MOI of 20. After 3 days of infection, the cells were inoculated into $6-\mathrm{cm}$ dishes at a density of $1 \times 10^{5}$ cells/dish. After $40 \mathrm{~h}$ of incubation, the cells in each well were harvested and the cell cycle was determined by propidium iodide (PI) staining before the cell density reached $80 \%$ confluency. Tests were performed in triplicate for each sample, and analyses were performed using a FACScan flow cytometer (Becton-Dickinson, San Jose, CA, USA) in accordance with the manufacturer's guidelines. 
A
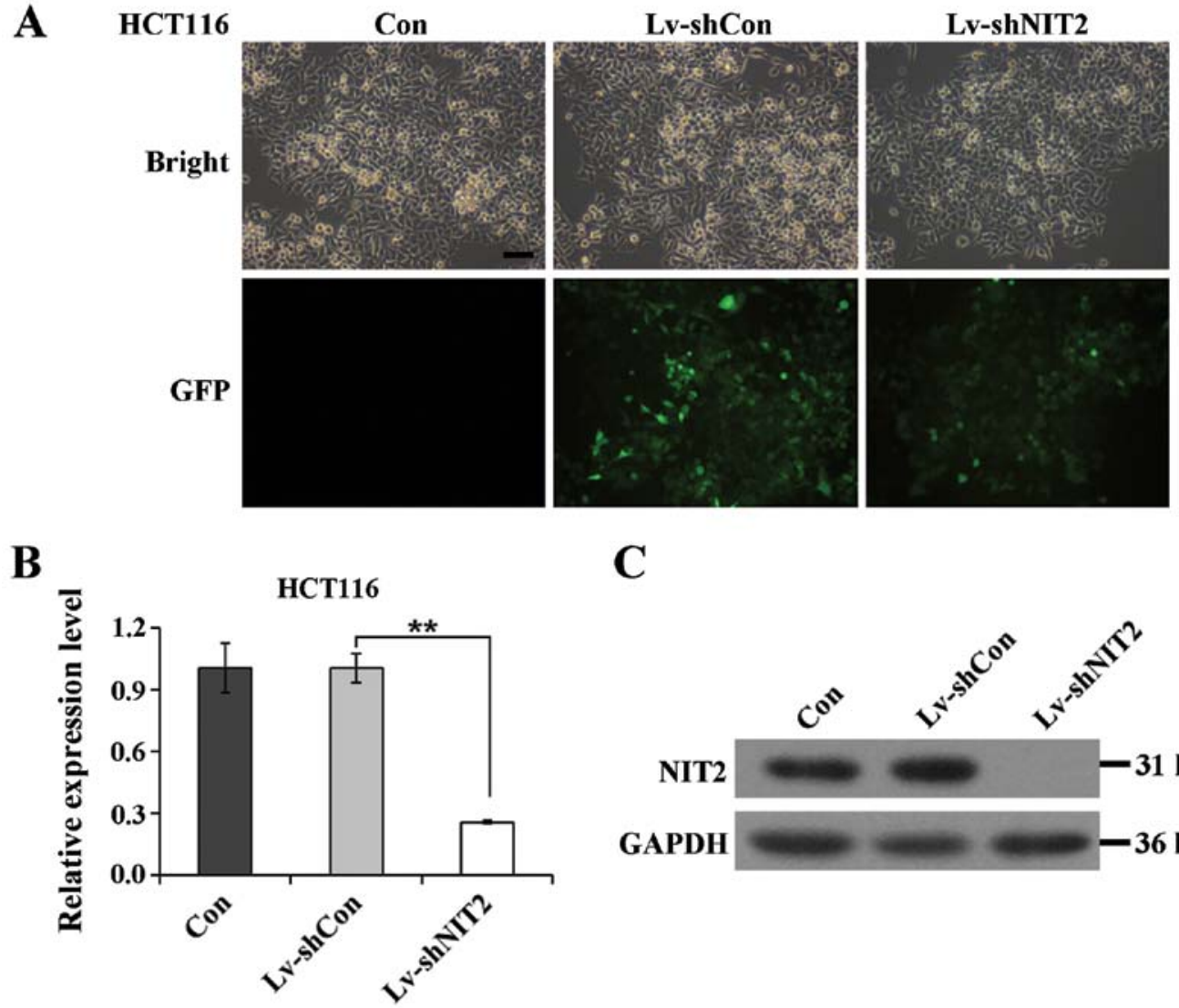

C

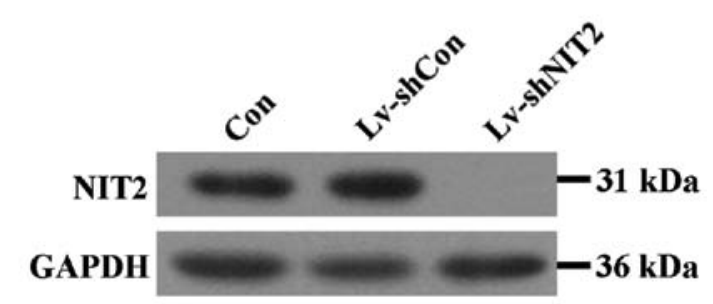

Figure 1. Knockdown efficiency of NIT2 by lentivirus infection in the colon cancer cells, HCT116. (A) Microscopic images of colon cancer cells infected with lentivirus at a multiplicity of infection (MOI) of 15 (scale bar, $50 \mu \mathrm{m}$ ). Visible green fluorescence protein (GFP) expression proved that $>80 \%$ of the cells were successfully infected. (B) qPCR analysis of NIT2 knockdown efficiency in HCT116 cells. The mRNA expression of NIT2 was significantly suppressed when the cells were infected with Lv-shNIT2. (C) Western blot analysis of NIT2 knockdown efficiency. ${ }^{* *} \mathrm{P}<0.01$.

Intracellular signaling array. Intracellular signaling array was carried out as previously described (16). The HCT116 cell lysates were analyzed using the PathScan Intracellular Signaling Array kit (Cat. no. 7323; Cell Signaling Technology) for the simultaneous detection of 18 important and wellcharacterized phosphorylated signaling molecules. The lysate of HCT116 was diluted to $200 \mathrm{mg} / \mathrm{ml}$, and $75 \mathrm{ml}$ of lysate was added to nitrocellulose-coated glass slides pre-coated with the primary antibodies. The plate was incubated overnight at $4^{\circ} \mathrm{C}$, followed by exposure to the detection antibody cocktail for $1 \mathrm{~h}$ at room temperature. HRP-conjugated secondary antibody was then added and the plate was incubated for $30 \mathrm{~min}$ at room temperature. Substrate was then added and chemiluminescent signals were detected to screen and compare the differences between cell lysates infected with Lv-shCon and Lv-shNIT2.

Statistical analysis. All statistical analyses were performed using SPSS version 13.0 software. The differences between groups were compared using the Student's test, and data are expressed as the means $\pm \mathrm{SD}$ of 3 independent experiments. A value of $\mathrm{P}<0.05$ was considered to indicate a statistical significant difference.

\section{Results}

Efficacy of lentivirus-mediated RNAi targeting of NIT2. In the present study, we constructed both a control lenti- virus (Lv-shCon) and a specific NIT2-targeting lentivirus (Lv-shNIT2). The HCT116 cells were cultured and infected with Lv-shCon or Lv-shNIT2. Non-infected cells were deemed as the negative control (Con). To demonstrate the infection efficiency, we employed a GFP tag which was embedded in the lentivirus to provide visualized confirmation. As shown in Fig. 1A, $>80 \%$ of the colon cancer cells were GFP-positive, indicating that the transfection rate was satisfactory. To further investigate the efficiency of the NIT2 knockdown, we examined the expression levels of NIT2 in the colon cancer cells following lentivirus infection. The non-silencing lentivirus encoded with the irrelevant sequence had a negligible effect on NIT2 expression, but the NIT2-silencing lentivirus markedly downregulated the mRNA expression of NIT2 by $74.6 \%$ (Fig. 1B). We further investigated the protein expression level following infection with the constructed lentiviruses. According to the NCBI gene database, the human NIT2 gene encodes a deduced protein comprising of 276 amino acids, with a calculated molecular mass of $30.6 \mathrm{kDa}$. As is shown in Fig. 1C, the protein expression of NIT2 in the HCT116 cells was noticeably depleted. Taken together, the lentiviruses we constructed conferred us an efficient vector system to knock down NIT2 expression in colon cancer cells.

Depletion of NIT2 markedly inhibits the proliferation of colon cancer cells. To better understand the role of NIT2 in colon cancer tumorigenesis, we examined the variation tendency of 
A

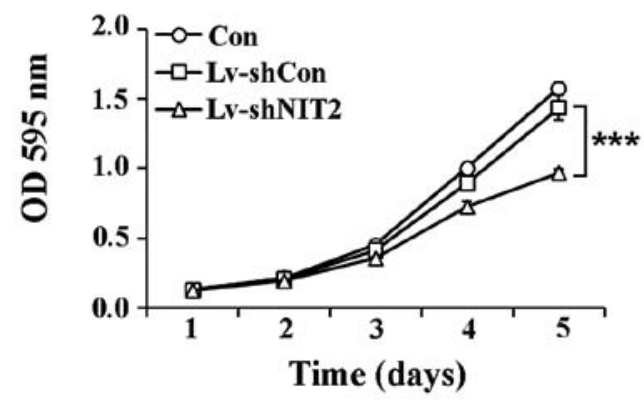

C

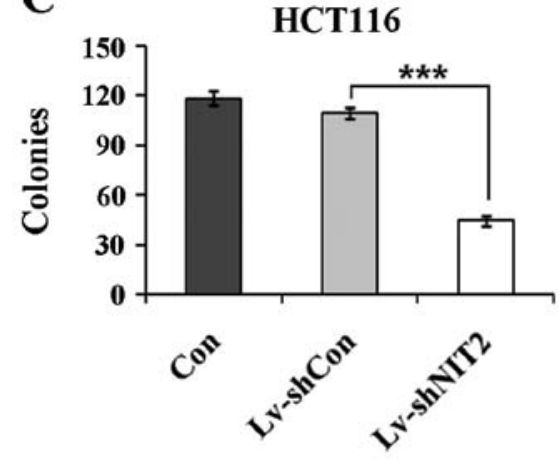

B

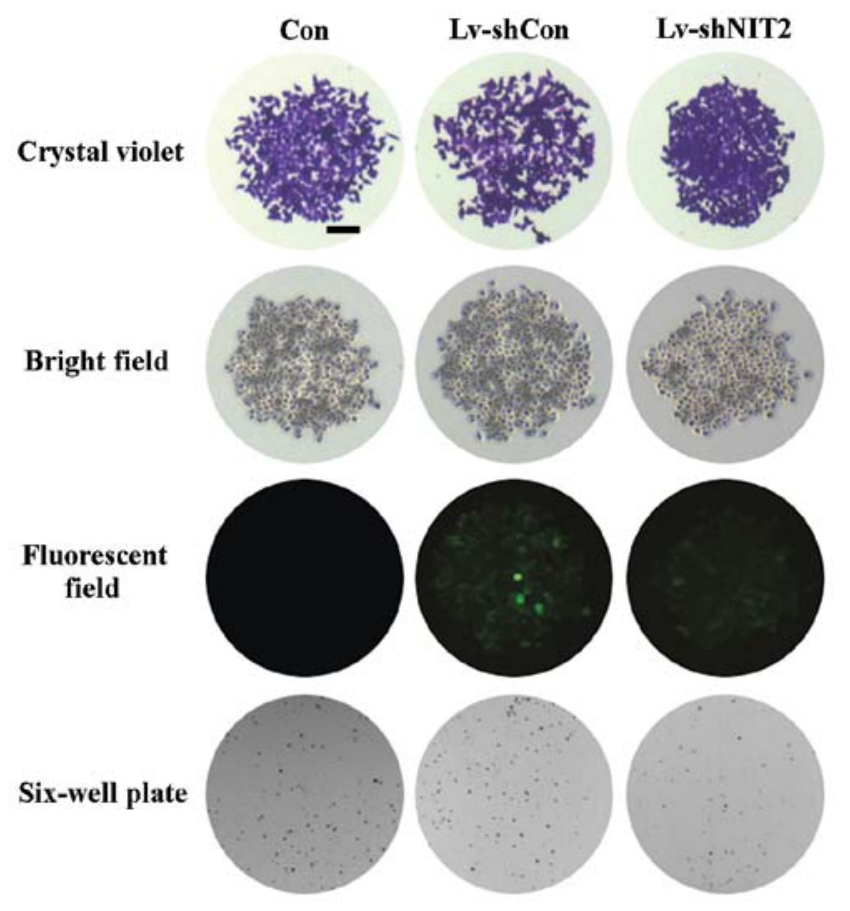

Figure 2. Depletion of NIT2 suppresses the proliferation and colony formation capacity of HCT116 cells. (A) The monolayer growth rates of HCT116 cells from triplicate groups (Con, Lv-Con and Lv-shNIT2) were determined by MTT assay. (B) Representative microscopic images of colonies were stained by crystal violet (scale bar, $125 \mu \mathrm{m}$ ). The full vision of the 6-well plate under a microscope showed a significant inhibition of colony formation in the Lv-shNIT2 group. (C) Statistical analysis of the number of colonies with $>50$ single cells stained with crystal violet. ${ }^{* * *} \mathrm{P}<0.001$.

A

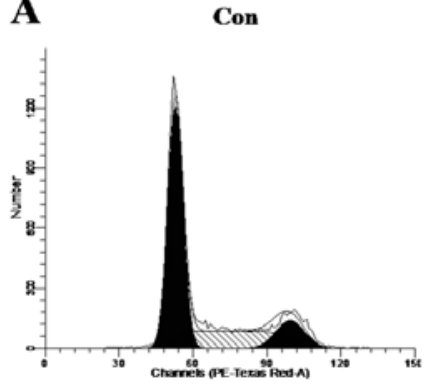

Lv-shCon

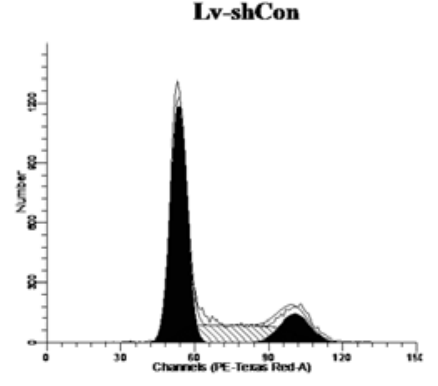

Lv-shNIT2

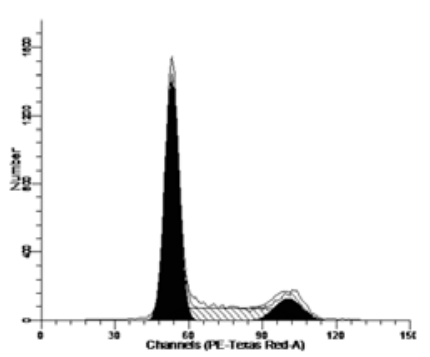

B

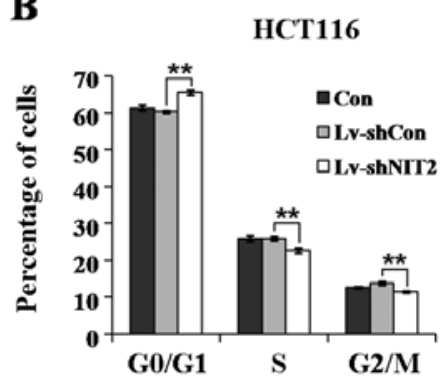

Figure 3. Downregulation of NIT2 induces G0/G1 phase cell cycle arrest in the HCT116 cells. (A) Cell cycle distribution of HCT116 cells was analyzed by flow cytometry. (B) The population of cells in the G0/G1 phase was markedly increased, accompanied by a noticeable reduction in the number of cells in the $\mathrm{S}$ and $\mathrm{G} 2 / \mathrm{M}$ phase. ${ }^{* *} \mathrm{P}<0.01$.

cell proliferation following lentivirus infection. We used MTT assay due to its testing sensitivity and dynamic range and examined the cell proliferation rate after 5 days of incubation. As illustrated by the line chart in Fig. 2A, the non-silenced cells showed no obvious difference compared with the control cells (Lv-shCon vs. Con); however, a significant inhibition of cell proliferation was observed in the NIT2-silenced cells (Lv-shNIT2 vs. Lv-shCon; P<0.001).

We subsequently cross evaluated the colony formation capacity of the HCT116 cells. As shown in Fig. 2B and C, the downregulation of NIT2 induced a significant decrease in the colony formation ability of the cells. The colonies were markedly smaller and the colony numbers were statistically fewer compared with the control cells $(\mathrm{P}<0.001)$, whereas there were no noticeable differences between the non-silenced cells and the control cells. Collectively, the knockdown of NIT2 by RNAi markedly suppressed the proliferation and colony formation ability of the colon cancer cells.

Cell cycle arrest is induced by the downregulation of NIT2. To examine whether the knockdown of NIT2 suppresses the growth of colon cancer cells through the direct regulation of the cell cycle, the effects of the suppression of NIT2 on the cell cycle distribution were examined. We explored the alterations in cell cycle distribution when the HCT116 cells were infected with the NIT2-silencing lentivirus. As illustrated in Fig. 3, the distribution of cells in the cell cycle (G0/G1, S and $\mathrm{G} 2 / \mathrm{M}$ phase) varied significantly between the 3 groups (Con, 


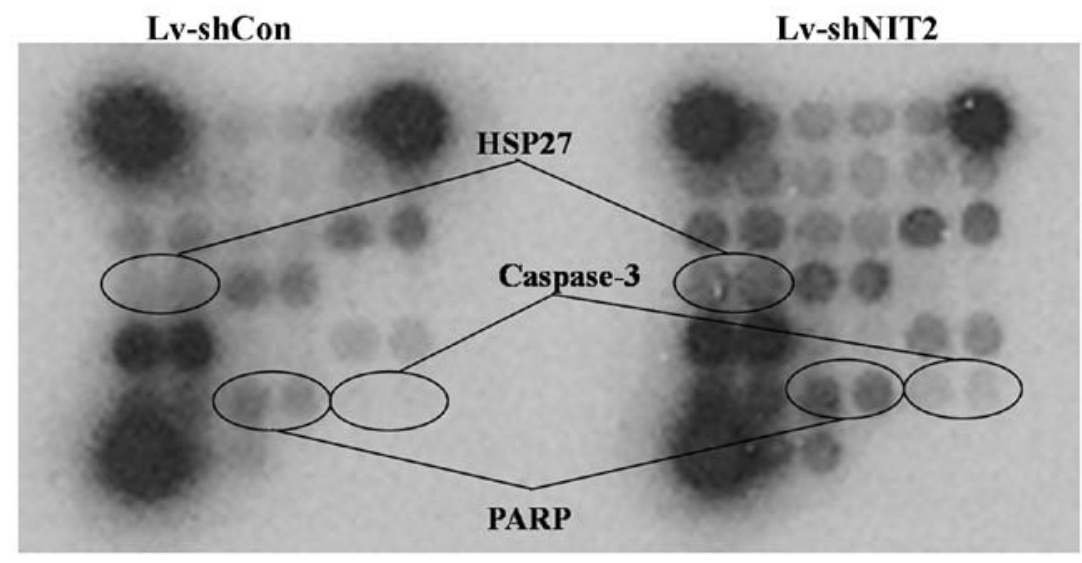

Figure 4. Chemiluminescent array shows the phosphorylation/cleavage status of 18 important signaling proteins in HCT116 cells. Modulation of NIT2 was associated with several important apoptotic signaling pathways in the HCT116 cells. The levels of cleaved PARP at Asp214 and caspase-3 at Asp175 were significantly elevated, accompanied by an increase of phosphorylated HSP27 (phosphor-site at Ser78).

Lv-shCon and Lv-shNIT2). In contrast to the control group, the cells infected with Lv-shNIT2 were mostly distributed in the $\mathrm{G} 0 / \mathrm{G} 1$ phase $(65.61 \%)$ and fewer cells were distributed in the $S$ phase (22.78\%) and the $\mathrm{G} 2 / \mathrm{M}$ phase (11.61\%), indicating that the cell cycle was arrested in the G0/G1 phase. Our results demonstrated that treatment with Lv-shNIT2 markedly induced cell cycle arrest $(\mathrm{P}<0.01)$; however, the cells infected with the non-silencing lentivirus did not show any significant differences compared with the non-infected cells (Lv-Con vs. Con). These findings are in agreement with those observed for cell growth, which suggests that NIT2 modulates colon cancer cell growth by controling the cell cycle.

Cell cycle arrest and inhibition of proliferation induced by the depletion of NIT2 are associated with caspase-3 and PARP signaling. The PathScan Intracellular Signaling Array kit was used to monitor the expression of 18 signaling molecules that are phosphorylated or cleaved in response to signal transduction pathway activation. Caspase-3 is a critical executor of apoptosis. Caspase-3 is activated by endoproteolytic cleavage at Asp175 and exerts its pro-apoptotic activity through the cleavage of multiple cellular targets (17-20). PARP, an enzyme that is involved in DNA repair, is one of the main substrates of activated caspase-3 $(21,22)$. Increased levels of cleaved caspase- 3 and cleaved PARP are reliable indicators of apoptosis (23). Heat shock protein 27 (HSP27), phosphorylated at Ser78 within the p38 MAPK pathway, is a mediator of cell stress that confers resistance to adverse environmental changes (24). In the present study, we demonstrated that when the colon cancer cells, HCT116, were treated with NIT2silencing lentivirus, the cleavage of PARP and caspase- 3 were noticeably increased, accompanied with an intracellular increase of phosphorylated HSP27 (Fig. 4).

\section{Discussion}

In mammals there are 2 metabolism routes for glutamine. One is catalyzed by glutaminases $\mathrm{K}$ and $\mathrm{L}$, followed by the conversion of glutamate to $\alpha$-ketoglutarate by transamination or by the glutamate dehydrogenase reaction, whereas the glutaminase II pathway employs $\omega$-amidase catalyzed hydrolysis reaction from $\alpha$-ketoglutamate (KGM) to $\alpha$-ketoglutarate (25-34). The glutaminase II pathway is one of the clinical interests, particularly in certain inborn errors of metabolism and cancers (35-38). This has been strengthened by the finding of NIT2, which was demonstrated to be a putative tumor suppressor (11) and identical to $\omega$-amidase $(28,29,39)$. NIT2/ $\omega$-amidase plays a crucial role in glutamine metabolism. However, its role in human colon cancer remains unclear.

In the present study, we examined the biological role of NIT2 in cell growth through an RNAi lentivirus system using the colon cancer cell line, HCT116, in vitro. We demonstrated that the downregulation of NIT2 suppressed cell proliferation and colony formation. Moreover, cell cycle arrest was observed by FACS analysis and apoptotic signaling markers were detected by an intracellular signaling array. The significant alterations in cell proliferation and cell cycle progression prove that NIT2 plays a pivotal role in the growth of colon cancer cells, which is in alignment with an increase in HSP27 phosphorylation at residue Ser78 and the cleavage of caspase-3 at residue Asp175 and PARP at residue Asp214.

Semba et al (40) reported that aberrant NIT1 expression induced caspase-dependent apoptosis. In addition, an apoptosis-inducing effect of NIT1 in plant wound and herbicide-induced cell death has been proposed (41). In contrast to NIT1, much less is known about the biological function of NIT2 in humans. To the best of our knowledge, this is the first study to prove that the depletion of NIT2 induces apoptosis through the caspase- 3 and PARP pathways. Caspase- 3 is a member of the cysteine-aspartic acid protease family (42). It interacts with caspase- 8 and caspase- 9 proteins and can trigger the sequential activation of cell apoptosis. Caspase-3 zymogen has virtually no activity until it is cleaved at Asp175 by an inhibitor caspase (43). Together with its main substrate PARP, both of them in the activated format can be deemed as reliable indicators of apoptosis (23).

Looking back to the observation of cell cycle arrest, all these data demonstrate that the depletion of NIT2 may not only inhibit colon cancer cell growth, but may also promote the apoptosis of cancer cells. The biochemical significance of NIT2 has been demonstrated in the present study, indicating 
that the depletion of NIT2 in colon cancer cells leads to a significant reduction in cell growth. Therefore, NIT2 may be a promising therapeutic target in colon cancer and may also be an alternative option for the treatment of colon cancer.

\section{References}

1. Merika E, Saif MW, Katz A, Syrigos K and Morse M: Colon cancer vaccines: an update (Review). In Vivo 24: 607-628, 2010.

2. Jemal A, Siegel R, Ward E, Hao Y, Xu J and Thun MJ: Cancer statistics, 2009. CA Cancer J Clin 59: 225-249, 2009.

3. Cunningham D, Atkin W, Lenz HJ, et al: Colorectal cancer Lancet 375: 1030-1047, 2010

4. Fearon ER: Molecular genetics of colorectal cancer. Annu Rev Pathol 6: 479-507, 2011

5. Bartling D, Seedorf M, Mithofer A and Weiler EW: Cloning and expression of an Arabidopsis nitrilase which can convert indole3 -acetonitrile to the plant hormone, indole-3-acetic acid. Eur J Biochem 205: 417-424, 1992.

6. Pace HC and Brenner C: The nitrilase superfamily: classification, structure and function. Genome Biol 2: REVIEWS0001, 2001.

7. Horst RJ, Zeh C, Saur A, Sonnewald S, Sonnewald U and Voll LM: The Ustilago maydis Nit2 homolog regulates nitrogen utilization and is required for efficient induction of filamentous growth. Eukaryot Cell 11: 368-380, 2012.

8. Pace HC, Hodawadekar SC, Draganescu A, et al: Crystal structure of the worm NitFhit Rosetta Stone protein reveals a Nit tetramer binding two Fhit dimers. Curr Biol 10: 907-917, 2000.

9. Pekarsky Y, Campiglio M, Siprashvili Z, et al: Nitrilase and Fhit homologs are encoded as fusion proteins in Drosophila melanogaster and Caenorhabditis elegans. Proc Natl Acad Sci USA 95: 8744-8749, 1998.

10. Barglow KT, Saikatendu KS, Bracey MH, et al: Functional proteomic and structural insights into molecular recognition in the nitrilase family enzymes. Biochemistry 47: 13514-13523, 2008.

11. Lin $\mathrm{CH}$, Chung MY, Chen WB and Chien $\mathrm{CH}$ : Growth inhibitory effect of the human NIT2 gene and its allelic imbalance in cancers. FEBS J 274: 2946-2956, 2007.

12. Kim DH, Behlke MA, Rose SD, Chang MS, Choi S and Rossi JJ: Synthetic dsRNA Dicer substrates enhance RNAi potency and efficacy. Nat Biotechnol 23: 222-226, 2005.

13. Guo W, Zhang Y, Chen T, et al: Efficacy of RNAi targeting of pyruvate kinase M2 combined with cisplatin in a lung cancer model. J Cancer Res Clin Oncol 137: 65-72, 2011.

14. Yang H, He X, Zheng Y, et al: Down-regulation of asparagine synthetase induces cell cycle arrest and inhibits cell proliferation of breast cancer. Chem Biol Drug Des 84: 578-584, 2014.

15. Shao X, Liu Y, Huang H, Zhuang L, Luo T and Ge X: Downregulation of $\mathrm{G}$ protein-coupled receptor 137 by RNA interference inhibits cell growth of two hepatoma cell lines. Cell Biol Int: Dec 9, 2014 (Epub ahead of print).

16. Miyake M, Goodison S, Urquidi V, Gomes Giacoia E and Rosser CJ Expression of CXCL1 in human endothelial cells induces angiogenesis through the CXCR2 receptor and the ERK1/2 and EGF pathways. Lab Invest 93: 768-778, 2013.

17. Boatright KM and Salvesen GS: Mechanisms of caspase activation. Curr Opin Cell Biol 15: 725-731, 2003.

18. Shi Y: Caspase activation: revisiting the induced proximity model. Cell 117: 855-858, 2004.

19. Kurokawa M and Kornbluth S: Caspases and kinases in a death grip. Cell 138: 838-854, 2009.

20. Brognard $\mathbf{J}$ and Hunter T: Protein kinase signaling networks in cancer. Curr Opin Genet Dev 21: 4-11, 2011.

21. Isabelle M, Moreel X, Gagne JP, et al: Investigation of PARP-1, PARP-2, and PARG interactomes by affinity-purification mass spectrometry. Proteome Sci 8: 22, 2010.
22. Piskunova TS, Yurova MN, Ovsyannikov AI, et al: Deficiency in poly(ADP-ribose) polymerase-1 (PARP-1) accelerates aging and spontaneous carcinogenesis in mice. Curr Gerontol Geriatr Res: 754190, 2008.

23. Yu SW, Andrabi SA, Wang H, et al: Apoptosis-inducing factor mediates poly(ADP-ribose) (PAR) polymer-induced cell death. Proc Natl Acad Sci USA 103: 18314-18319, 2006.

24. Parcellier A, Schmitt E, Gurbuxani S, et al: HSP27 is a ubiquitin-binding protein involved in I-kappaBalpha proteasomal degradation. Mol Cell Biol 23: 5790-5802, 2003.

25. Meister A and Otani TT: $\omega$-Amide and $\omega$-amino acid derivatives of alpha-ketoglutaric and oxalacetic acids. J Biol Chem 224: $137-148,1957$.

26. Meister A, Levintow L, Greenfield RE and Abendschein PA: Hydrolysis and transfer reactions catalyzed by omega-amidase preparations. J Biol Chem 215: 441-460, 1955.

27. Meister A: Preparation of enzymatic reactions of the keto analogues of asparagine and glutamine. J Biol Chem 200: 571-589, 1953.

28. Krasnikov BF, Chien CH, Nostramo R, et al: Identification of the putative tumor suppressor Nit2 as omega-amidase, an enzyme metabolically linked to glutamine and asparagine transamination. Biochimie 91: 1072-1080, 2009.

29. Jaisson S, Veiga-da-Cunha M and Van Schaftingen E: Molecular identification of omega-amidase, the enzyme that is functionally coupled with glutamine transaminases, as the putative tumor suppressor Nit2. Biochimie 91: 1066-1071, 2009.

30. Hersh LB: Rat liver-amidase. Kinetic evidence for an acylenzyme intermediate. Biochemistry 11: 2251-2256, 1972.

31. Hersh LB: Rat liver omega-amidase. Purification and properties. Biochemistry 10: 2884-2891, 1971.

32. Duffy TE, Cooper AJ and Meister A: Identification of alphaketoglutaramate in rat liver, kidney, and brain. Relationship to glutamine transaminase and $\omega$-amidase activities. J Biol Chem 249: 7603-7606, 1974

33. Cooper AJ and Meister A: Isolation and properties of a new glutamine transaminase from rat kidney. J Biol Chem 249: 2554-2561, 1974.

34. Cooper AJ: Asparagine transaminase from rat liver. J Biol Chem 252: 2032-2038, 1977.

35. Kuhara T, Inoue Y, Ohse M,Krasnikov BF and Cooper AJ: Urinary 2-hydroxy-5-oxoproline, the lactam form of $\alpha$-ketoglutaramate, is markedly increased in urea cycle disorders. Anal Bioanal Chem 400: 1843-1851, 2011.

36. Szeliga M and Obara-Michlewska M: Glutamine in neoplastic cells: focus on the expression and roles of glutaminases. Neurochem Int 55: 71-75, 2009.

37. Wang JB, Erickson JW, Fuji R, et al: Targeting mitochondrial glutaminase activity inhibits oncogenic transformation. Cancer Cell 18: 207-219, 2010

38. Erickson JW and Cerione RA: Glutaminase: a hot spot for regulation of cancer cell metabolism? Oncotarget 1: 734-740, 2010.

39. Krasnikov BF, Nostramo R, Pinto JT and Cooper AJ: Assay and purification of omega-amidase/Nit2, a ubiquitously expressed putative tumor suppressor, that catalyzes the deamidation of the $\alpha$-keto acid analogues of glutamine and asparagine. Anal Biochem 391: 144-150, 2009.

40. Semba S, Han SY, Qin HR, et al: Biological functions of mammalian Nit1, the counterpart of the invertebrate NitFhit Rosetta stone protein, a possible tumor suppressor. J Biol Chem 281: 28244-28253, 2006.

41. Cutler SR and Somerville CR: Imaging plant cell death: GFP-Nit1 aggregation marks an early step of wound and herbicide induced cell death. BMC Plant Biol 5: 4, 2005.

42. Alnemri ES, Livingston DJ, Nicholson DW, et al: Human ICE/ CED-3 protease nomenclature. Cell 87: 171, 1996.

43. Walters J, Pop C, Scott FL, et al: A constitutively active and uninhibitable caspase-3 zymogen efficiently induces apoptosis. Biochem J 424: 335-345, 2009. 